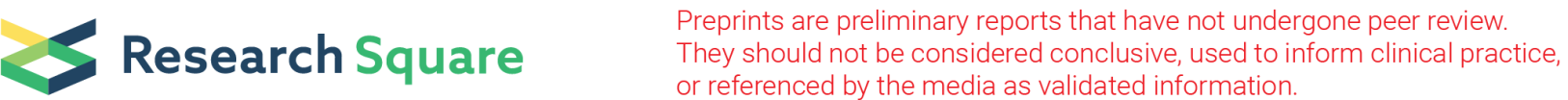

\section{Clinical Laboratory Blood Biomarker-Based Nomograms for Prediction of Lymph Node Metastasis and Prognosis in Resectable Esophagogastric Junction Adenocarcinoma: A Retrospective Diagnostic Study}

Can-Tong Liu

Cancer Hospital of Shantou University Medical College

Chao-Qun Hong

Cancer Hospital of Shantou University Medical College

Hai-Peng Guo

Cancer Hospital of Shantou University Medical College

Xu-Chun Huang

Cancer Hospital of Shantou University Medical College

Hong Guo

Cancer Hospital of Shantou University Medical College

Xin-Yi Huang

Shantou University Medical College

Ling-Yu Chu

Cancer Hospital of Shantou University Medical College

Yi-Wei Lin

Cancer Hospital of Shantou University Medical College

Lai-Feng Wei

Cancer Hospital of Shantou University Medical College

Yu-Hui Peng

Cancer Hospital of Shantou University Medical College

Yi-Wei Xu ( $\nabla$ yiwei512@126.com )

Cancer Hospital of Shantou University Medical College

\section{Research Article}

Keywords: Esophagogastric junction adenocarcinoma, Clinical laboratoryblood biomarkers, nomogram, lymph node metastasis, prognosis 
Posted Date: February 24th, 2022

DOI: https://doi.org/10.21203/rs.3.rs-1383267/v1

License: (c) (i) This work is licensed under a Creative Commons Attribution 4.0 International License. Read Full License 


\section{Abstract \\ Background}

Esophagogastric junction adenocarcinoma (EJA) lacks blood-based predictive biomarkers for lymph node status and prognosis. We aimed to build nomograms with the preoperative blood biomarkers to predict lymph node metastasis (LNM) and prognosis in resectable EJA.

\section{Methods}

In this study, EJA patients who underwent surgery were collected from the Cancer Hospital of Shantou University Medical College between January 2010 and December 2017. The patients were divided into training cohort $(n=465)$ and validation cohort $(n=289)$ chronologically. 52 candidate biomarkers for nomogram construction included sociodemographic data and preoperative clinical laboratory blood baseline data. Lasso regression was used to build LNM prediction nomogram (LNM nomogram). Univariate and multivariate Cox regression analysis was applied to select factors for overall survival prediction nomogram (OS nomogram).

\section{Results}

As a result, for predicting LNM, the LNM nomogram containing seven biomarkers showed good discrimination and calibration ability, with C-indexes of 0.684 and 0.630 in the training and validation cohorts, respectively. Moreover, the OS nomogram for survival prediction composed of 12 factors yielded C-indexes of 0.652 and 0.663 in the training and validation cohorts, respectively. Kaplan-Meier survival analysis showed patients with high OS nomogram scores had worse 5-year OS than those with low scores (training cohort: $59.6 \%$ vs. $26.2 \%$; validation cohort: $58.6 \%$ vs. $38.6 \%$; all $p<0.0001$ ).

\section{Conclusions}

In conclusion, these two nomograms constructed based on the clinical laboratory blood biomarkers are potential prediction models for lymph node status and survival of resectable EJA.

\section{Background}

Gastric and esophageal cancers rank fourth and sixth in the mortality of cancers worldwide, respectively(1). Esophagogastric junction adenocarcinoma (EJA), first described by Siewert in 1998, is a type of malignant tumor with center located at a 10-centimeter distance between esophagus and stomach (2). The prevalence of EJA is high in areas where the incidence of esophageal squamous cell cancer is also high (3). Compared to the 5 -year overall survival rates (OS) of less than $50 \%$ for patients 
with advanced gastric or esophageal adenocarcinoma after surgery, it was reported that the 5-year OS of advanced EJA patients receiving surgery was even poorer, less than $30 \%$ (4).

In the clinical practice, cancer patients with positive lymph node metastasis (LNM) are usually required to receive neoadjuvant chemotherapy or radiotherapy. Thus, accurate LNM prediction is a prerequisite for individualized precision therapy of EJA. Computed tomography provides some pre-treatment information for LNM, with the diagnostic standard for positive LNM of enlarged lymph node over 10 millimeters (5). However, the determination of LNM by computed tomography was limited, with the reported accuracy rate varying from $25-78 \%$ (6). Another study also found that only $9.3 \%$ patients met this standard after pathological definition (7). On the other hand, one of the most vital concerns is to recognize the prognosis of EJA, especially before receiving treatments. Recently, some preoperative blood biomarkers, like platelets, CA19-9, neutrophil-lymphocyte ratio, and platelet-lymphocyte ratio, were also found to be in connection with LNM in different cancers (8-11). Moreover, other publications identified some novel independent blood-based prognostic factors in EJA patients, such as prognostic nutritional index (PNI), systemic immune-inflammation index (SII), C-reactive protein/albumin ratio (CRP/ALB), and so on (1214). However, the obvious shortages of these studies could be listed as lack of combined detection of biomarkers and limited samples of EJA. Furthermore, none of them focused on resectable EJA. Therefore, it is necessary and feasible to find an objective and vigilant significant method based on the combined detection of blood-related biomarkers to predict lymph node status and survival for EJA.

In recent years, nomogram has been used as a tool in predicting lymph node status and cancer outcomes (15). A study constructed a radiomics nomogram incorporating both radiomics signature and clinical risk factors to facilitate the preoperative prediction of LNM in patients with colorectal cancers (16). Increasing reports show that some novel constructed nomograms might be more accurate than the TNM staging system in cancer prognosis prediction, such as lung, gastric, breast cancers and so on (17-19). Since no blood biomarkers based nomogram has been built for EJA until now, in the present study, we constructed and validated nomograms based on blood indicators for prediction of LNM and survival in resectable EJA.

\section{Methods}

\section{Enrolling participants}

In this retrospective study, we enrolled EJA patients who received surgery resection at their first impatient time in Cancer Hospital of Shantou University Medical College from December 2003 to December 2017. EJA was diagnosed based on gastroscopy and computed tomography, and further confirmed by pathology after surgery. The participants from December 2003 to December 2010 were assigned into training cohort and those from January 2011 to December 2017 were into validation cohort. The enrolled patients for analysis were those without previous history of other malignant tumors or tumor-related treatments, and with complete clinicopathological and follow-up data. Patients suffered from renal failure, autoimmune diseases or infection diseases were excluded for analysis. Those who had a fever at 
the time of blood drawing were also excluded. Patients with unresectable tumors due to serious abdominal adhesion, large tumor infiltration range or other factors were also excluded, as well as the pathologically determined liver or lung metastasis. The ethical approval was obtained through the ethics committee of Cancer Hospital of Shantou University Medical College who surrendered the need for patient consent when the individual consents were not identified. And the research was conducted according to Declaration of Helsinki.

\section{Data collection}

We obtained the data comprised of relevant information on sociodemographic data (gender, age, body mass index, family tumor history, smoking history, and alcohol consumption history), pathological data (tumor size, invasive depth, lymph node status, and Siewert's classification) and preoperative clinical laboratory blood data within one week (complete blood cell count, coagulation, and biochemical parameters). The complete blood cell count included white blood cells (WBC), red blood cells (RBC), hemoglobin (HGB), hematocrit (HCT), mean corpuscular volume (MCV), mean corpuscular hemoglobin $(\mathrm{MCH})$, mean corpuscular hemoglobin concentration $(\mathrm{MCHC})$, platelets (PLT), lymphocyte count (LY), monocytes count (MO) and neutrophil count (NE). The coagulation parameters contained thrombin time (TT) and fibrinogen (Fib). The biochemical biomarkers included creatine kinase (CK), lactate dehydrogenase $(\mathrm{LDH})$, hydroxybutyrate dehydrogenase $(\mathrm{HBDH})$, aspartate aminotransferase (AST), alanine transaminase (ALT), total protein (TP), albumin (ALB), globin (GLB), total bilirubin (TBIL), direct bilirubin (DBIL), indirect bilirubin (IBIL), glucose (Glu), creatinine (Cr), uric acid (UA), immunoglobulin $A$ ( $\lg A)$, immunoglobulin $G(\operatorname{lgG})$, immunoglobulin $M(\operatorname{lgM})$, complement 3 (C3), complement factor $B(C F B)$, and $\mathrm{C}$-reactive protein (CRP). Some combined indicators reported in our previous review were also listed in this study, containing neutrophil-to-lymphocyte ratio (NLR), platelet-to-lymphocyte ratio (PLR), lymphocyte-to-monocyte ratio (LMR), albumin-to-globin ratio (AGR), prognostic nutritional index (PNI), and systemic immune-inflammation score (SII) (20).

The TNM stages of the enrolling patients were defined according to the eighth edition of American Joint Committee on Cancer Staging Manual (21). EJA, whose tumor centers were less than 2 centimeters away the esophagogastric junction, were staged as esophageal carcinomas, while those with over 2 centimeters were staged as gastric cancers. The latter ones were also known as gastric cardia cancers or Siewert's type III EJA. All the enrolling patients received the follow-up. OS was defined from the date of receiving surgery to any form of death and the data were censored for patients who were alive at the last follow-up of December 2019.

\section{Hematological score building and nomogram for LNM}

The individual cut-off values of sociodemographic data and preoperative clinical laboratory blood data in the training cohort for determining positive LNM were defined by maximizing the absolute Youden index in the receptor operating characteristic curves. Then we changed the continuous variables into dichotomous variables based on the above cut-off values. Lasso regression, which was created to avoid overfitting or underfitting, was used to select the most useful variables in the training cohort (22). Blood 
score was calculated by a linear combination of the selected variables weighted by their respective nonzero coefficients from Lasso regression. And we constructed a nomogram (named as "LNM nomogram") for LNM prediction based on the selected variables to be visualized.

\section{Nomogram for survival prediction}

In order to construct nomogram for predicting OS of patients, we modified the continuous variables, including sociodemographic data and preoperative clinical laboratory blood data, into dichotomous variables using the cut-off values calculated by survivalROC package in $\mathrm{R}$ version through maximizing the absolute Youden index. Then we built a nomogram (named as "OS nomogram") for predicting OS of EJA patients using the independent survival predictive factors obtained according to the results of univariate and multivariate Cox regression analysis.

\section{Assessment of the nomograms}

The Harrell's consistence index (C-index) was measured to access the discrimination performance of different nomograms. Bootstrap was used to evaluate the accuracy of the nomograms, and the calibration curves were created using the margin effect and the average prediction probability of the model. The improvement in predicting prognosis was assessed by net reclassification improvement (NRI) and integrated discrimination improvement (IDI). After calculating all patients' nomogram scores based on OS nomogram, we further used survivalROC package to identify the best cut-off value were defined by maximizing the absolute Youden index for distinguishing different prognostic risk of EJA patients.

\section{Statistical analysis}

All the statistical analyses were achieved using the following software: Microsoft Excel, IBM SPSS statistics 24.0 (SPSS Inc., Chicago, IL, USA), and R version 3.6.1 (http://www.R-project.org/). Lasso regression analysis was achieved by the g/mnet package in R. The nomograms and calibration curves were plotted by the rms package, and NRI and IDI were achieved by the survIDINR/ package. Survival curves were plotted using Kaplan-Meier survival analysis and compared by the log-rank test through the survminer packages. Statistical significance was set at $p$ lower than 0.05 (two-tailed) in this study.

\section{Results}

\section{Patients recruitment}

As shown in Fig. 1, from December 2003 to December 2017, there were total 754 EJA patients who met the enrolled standard in Cancer Hospital of Shantou University Medical College. From these, 465 patients were appointed to the training cohort, and the validation cohort was comprised of the other 289 patients (Table 1). 
Table 1. Characteristics of EJA Patients in the Training and Validation Cohorts

Training Cohort $(n=465)$

Negative lymph node status
Positive lymph node status

Validation Cohort $(n=289)$

$39.17 \pm 5.86$

Median OS

(months)

5-year OS (\%) $\quad 44.30 \pm 2.30$

3-year OS (\%) $\quad 51.60 \pm 2.30$

1 -year OS (\%) $\quad 83.90 \pm 1.70$

Age, mean $\pm \quad 60.14 \pm 8.26$

$S D$, years

Gender

Male

106

283

50

$p$

Negative

lymph node status

Positive lymph node status

$37.50 \pm 6.79$

$43.80 \pm 3.00$

$50.90 \pm 2.90$

- $\quad 82.77 \pm 2.20$

$61.32 \pm 7.95 \quad 0.097 \quad 63.03 \pm 7.18$

$64.07 \pm 7.39$

0.280

- $\quad 50.90 \pm 2.90$

Female

26

0.218

0.251

58

170

BMI, mean $\pm \quad 21.96 \pm 3.09$

$\mathrm{SD}, \mathrm{kg} / \mathrm{m}^{2}$

Tumor

0.893

0.398

Family

$\begin{array}{ccccc}\text { No } & 114 & 286 & 65 & 184 \\ \text { Yes } & 18 & 47 & 13 & 27\end{array}$

Smoking

0.371

0.203

History

$\begin{array}{lllll}\text { No } & 51 & 114 & 31 & 67 \\ \text { Yes } & 81 & 219 & 47 & 144\end{array}$

Drinking

0.385

0.274

History

$\begin{array}{lllll}\text { No } & 103 & 247 & 55 & 162 \\ \text { Yes } & 29 & 86 & 23 & 49\end{array}$

TNM stage

I (IA+IB+IC) 34

2

20

0

II (IIA+IIB) 74

38

37

21

III (IIIA+IIIB)

215

21

149


IV (IIIC+IVA)

Siewert's

Classification
78

0.005

41

$\begin{array}{lllll}\begin{array}{l}\text { Siewert's type } \\ \text { I/II }\end{array} & 90 & 180 & 57 & 115\end{array}$

$\begin{array}{lllll}\text { Siewert's type } & 42 & 153 & 21 & 96\end{array}$

Note: EJA: esophagogastric junction adenocarcinoma; OS: overall survival; SD: standard deviation; BMl: body mass index.

\section{H-score nomogram for LNM prediction}

The cut-off values of continuous variables changing into dichotomous ones for determining positive LNM were shown in Supplementary Table S1. Using the Lasso logistic regression, 52 variables including sociodemographic data and preoperative clinical laboratory blood biomarkers were reduced to seven potential predictors in the training cohort with nonzero coefficients with the $\lambda$ value of 0.049 (Fig. 2). These predictors were presented in the blood score calculation formula: Blood score $=0.7800360563+$ PLT $\times 0.2457506084-$ NE\% $\times 0.0278343059-$ Fib $\times 0.0196964930+$ ALP $\times 0.0802384761-$ Glu $\times$ $0.0130250072+\mathrm{CRP} / \mathrm{ALB} \times 0.1686066797+\mathrm{SII} \times 0.0003642568\left(\mathrm{PLT}>290.50 \times 10^{9} / \mathrm{I}\right.$ was defined as 1 point, while $<290.50 \times 10^{9} / \mathrm{I}$ was defined as 0 point. Similarly, NE\% $>47.85 \%$, Fib $>2.64 \mathrm{~g} / \mathrm{l}, \mathrm{ALP}>123.35$ $\mathrm{U} / \mathrm{l}, \mathrm{Glu}>5.00 \mathrm{mmol} / \mathrm{I}, \mathrm{CRP} / \mathrm{ALB}>0.04$, and $\mathrm{SII}>742.45$ were defined as 1 point). The $\mathrm{C}$-index of the blood score yielded 0.684 (95\% Cl: $0.629 \sim 0.738$ ) in the training cohort, and that in the validation cohort was 0.630 (95\% Cl: $0.556 \sim 0.703)$. We then constructed LNM nomogram based on these seven predictors (Fig. 3A). The calibration curves demonstrated that the nomogram-predicted probability of LNM were consistent with the actual LNM both in the training and validation cohorts (Fig. 3B and 3C).

\section{OS nomogram for survival prediction}

In the training cohort, the median survival time of resectable EJA patients was $39.17 \pm 5.86$ months with the 5 -year OS of $44.30 \%$, like those of the validation cohort (37.50 \pm 6.79 months with the 5 -year OS of $43.80 \%$, Table 1). After univariate Cox regression analysis, 17 biomarkers with $p$ value of less than 0.05 were selected for stepwise backward multivariate Cox analysis (Supplementary Table S2). And we finally determined twelve independent prognostic biomarkers, which were used to construct OS nomogram for survival prediction of EJA patients (Fig. 4).

\section{Evaluation of OS nomogram}

As shown in Table 2, the C-index of OS nomogram was 0.652 (95\% Cl: $0.620 ~ 0.684)$ in the training cohort, higher than that of TNM system $(0.623 ; 95 \% \mathrm{Cl}: 0.620 \sim 0.684 ; p=0.044)$. After combining OS 
nomogram with the TNM system, we observed that the C-index increased to 0.709 (95\% Cl: 0.680 $0.737)$, also higher than that of TNM system $(p<0.001)$. In the validation cohort, the C-index of combination improved to 0.704 (95\% Cl: $0.664 \sim 0.744$ ) when compared with TNM system alone ( $p<$ 0.001). Then we further applied NRI and IDI to evaluate the improvement of the combination of OS nomogram and TNM system compared with TNM system alone in predicting prognosis. Figure 5A demonstrated that the predictive accuracy of the combination was better than that of TNM system alone in predicting 5-year OS in the training cohort (NRI $=0.300,95 \% \mathrm{Cl}: 0.198 \sim 0.393, p<0.001 ; \mathrm{IDI}=0.114$, $95 \% \mathrm{Cl}: 0.072 \sim 0.164, p<0.001)$. Similar results were found in the validation cohort (Fig. 5B; NRI $=0.265$, $95 \% \mathrm{Cl}: 0.085 \sim 0.397, p=0.008 ; \mathrm{IDI}=0.054,95 \% \mathrm{Cl}: 0.016 \sim 0.109, p=0.004)$. Furthermore, the calibration curves showed that the nomogram-predicted probability of 5-year OS were consistent with the actual 5-year OS both in training and validation cohorts (Fig. 5C and 5D).

Table 2

The C-index of OS nomogram for predicting OS of EJA patients

\begin{tabular}{|lccc|}
\hline & C-index & $95 \% \mathrm{Cl}$ & $\boldsymbol{p}$ \\
\hline Training cohort & & & \\
\hline TNM system & 0.623 & $0.598 \sim 0.647$ & Ref. \\
\hline OS nomogram & 0.652 & $0.620 \sim 0.684$ & 0.044 \\
\hline OS nomogram + TNM system & 0.709 & $0.680 \sim 0.737$ & $<0.001$ \\
\hline Validation cohort & & & \\
\hline TNM system & 0.616 & $0.583 \sim 0.648$ & Ref. \\
\hline OS nomogram & 0.663 & $0.628 \sim 0.705$ & 0.006 \\
\hline OS nomogram + TNM system & 0.704 & $0.664 \sim 0.744$ & $<0.001$ \\
\hline Note: OS: overall survival; EJA: esophagogastric junction adenocarcinoma; Cl: confidence interval \\
\hline rOgnostic Stratification based On OS nomogram
\end{tabular}

In order to evaluate whether the OS nomogram could stratify the risk of EJA patients with different outcomes, we calculated the nomogram score of each patient according to the OS nomogram, and obtained the best cut-off value (344 scores) by using survivalROC package. From Fig. 5E and 5F, the OS nomogram could separate high-risk patients from low-risk patients, and patients with high scores had the worse 5-year OS than those with low scores (training cohort: $59.6 \%$ vs. $26.2 \%$; validation cohort: $58.6 \%$ vs. $38.6 \%$; All $p<0.0001$ ).

\section{Discussion}

Inflammation has been accepted as a hallmark of cancer progression and prognosis (23). In the constructed LNM nomogram for positive LNM prediction, more than half of the enrolled biomarkers were 
inflammation-related parameters, including NE\%, platelets, Fib, ALP, CRP/ALB and SII. Therefore, it highlighted the high correlation between inflammation and LNM. On the other hand, considering the poor survival of patients with EJA, some models based on hematological biomarkers were constructed to predict the survival of patients with EJA. For example, a baseline prognostic model containing ALP for patients with locally advanced or metastatic esophago-gastric cancers was constructed by London Royal Marsden Hospital, and validated by two independent randomized controlled trials (24-26). Another study from the Yale Cancer Center also built another model for patients with advanced gastric cancer or EJA using twelve indicators, among which there was seven blood-based biomarkers, including ALP (27). However, these studies were not directedly specifical at EJA patients, with the small sample size of EJA patients which weakened the power of model prediction evaluation. Furthermore, there was no study of nomogram targeted EJA patients receiving surgery resection. Thus, in this study, we established nomograms for survival prediction of EJA patients undergoing surgery with cohort validation. When we combined the OS nomogram with the traditional TNM system, it would improve the survival predictive ability.

In 1992, some researchers proposed that angiogenesis was closely related to the occurrence and metastasis of breast cancer, and platelet endothelial cell adhesion factor antibody, as an indicator of angiogenesis, could be used to evaluate LNM and prognosis of breast cancer patients (28). Platelet endothelial cell adhesion factor can be expressed in platelets, endothelial cells, and neutrophils. In previous published studies, PLT was also found to be associated with LNM of colon, lung and endometrial cancer $(8,29,30)$. However, in our previous review, most studies found that platelets were not associated with the prognosis of EJA patients (31). In our study, we found that PLT had a large positive weight in the LNM prediction nomogram constructed for EJA, and high platelet was an independent predictor of poor prognosis. It has been considered that platelet could affect the biological behavior of tumor cells, participate in tumor angiogenesis, affect tumor microenvironment, and protect circulating tumor cells from the attack of the immune system (32). Therefore, we speculate that platelets may play an important role in influencing LNM and prognosis of EJA patients.

We found that ALP was one of the significant indicators in the two nomograms constructed in our study, which also entered the models constructed by the Royal Marsden Hospital and the Yale University study. The increasing of ALP activity usually indicates the occurrence of biliary obstruction and tumor bone metastasis. Most of the previous studies have also proposed its correlation with tumor bone metastasis, including gastric, prostate, and esophageal cancer (33-35). Aminian et al. believed that esophageal cancer patients with high ALP level tended to have positive LNM (36). ALP was also reported to associated with the survival rate of solid cancers $(37,38)$. Namikawa et al. found that high ALP level was an independent and worse prognosis factor for patients with unresectable gastric cancer receiving chemotherapy (33). In our two nomograms, we found that EJA patients with high ALP were also prone to positive LNM and the prognosis of patients with high ALP was poor.

SIl is another biomarker in both nomograms. It was an indicator proposed in 2014 when discussing the variables for predicting the prognosis of hepatocellular carcinoma (39). Meta analysis found that high 
levels of SII were associated with increased risk of LNM in esophageal and gastric cancer $(40,41)$. In this study, we found that high preoperative SIl level was associated with LNM in EJA patients, but it was a protective factor for prognosis, which was not consistent with the previous study (13). It might be due to the different cutoff values in two nomograms. Therefore, in the future, the predictive value of SII in EJA should be validated in a larger sample size study.

Cancer can activate the immune system to produce antibodies against cancer. Many studies suggest that the detection of tumor antibody in blood can help to diagnose and judge the prognosis of cancers. Ishdorj et al. found that low level of IgG and high level of IgA were important independent prognostic biomarkers in chronic lymphoblastic leukemia (42). In addition to the antigen antibody system, there is a complement system. The overexpression of $\mathrm{C} 3$ can affect the progress of gastric cancer by activating JAK2/STAT3 signaling pathway, and reduce the 5-year OS of gastric cancer patients (43). Moreover, some studies found that the level of CFB in plasma of pancreatic cancer patients was twice higher than that of patients without pancreatic cancer, with the diagnostic efficiency of 0.958 , better than CA19-9 (0.833). And patients with high CFB had a shorter survival than patients with low CFB $(44,45)$. In this study, we also found that preoperative high levels of IgA, C3, CFB, and low levels of IgG were risk factors for poor prognosis of EJA patients, but further study was needed to explore the mechanisms.

As a retrospective study, there were some limitations. First, it was a single-center study, and studies from other institutes were needed to further validate our nomograms. Second, as the follow-up time of some patients in the validation cohort was less than 5 years, it may have a certain impact on the results. In future studies, we will further follow up these patients, in order to evaluate the OS nomogram more accurately for EJA prognosis prediction. Third, Tokunaga found that CA19-9 could serve as a useful prognostic biomarker in patients with EJA (46). However, in our study, most of the enrolled patients did not receive tumor biomarkers examination. If future study issued this problem, it might be helpful to provide more accurate prediction of EJA prognosis. The last but not least, two nomograms for LNM and prognosis prediction, respectively, appear to be complicated. However, since the natures of LNM and prognosis prediction are different, we believe that two nomograms would be more suitable for the request of precision diagnosis.

\section{Conclusions}

To sum up, we evaluated 52 biomarkers containing the preoperative clinical laboratory blood test results and sociodemographic indicators, and constructed LNM nomogram including seven indicators and OS nomogram including twelve indicators, which have the potential to predict LNM and prognosis of resectable EJA patients, respectively. These two nomograms both have good accuracy, and the results are consistent in the validation cohort. In the future, if further evaluation could be carried out and verified in more research centers, the nomograms we constructed would be expected to provide new suggestion for the individualized treatment options of EJA patients.

\section{Abbreviations}


EJA: Esophagogastric Junction Adenocarcinoma; LNM: lymph node metastasis; BMI: Body Mass Index; WBC: White Blood Cells; RBC: Red Blood Cells; HGB: Hemoglobin; HCT: Hematocrit; MCV: Mean Corpuscular Volume; MCH: Mean Corpuscular Hemoglobin; MCHC: Mean Corpuscular Hemoglobin Concentration; PLT: Platelets; NLR: Neutrophil-to-Lymphocyte ratio; PLR: Platelet-to-Lymphocyte ratio; LMR: Lymphocyte-to-Monocyte ratio; TT: Thrombin Time; Fib: Fibrinogen; CK: Creatine Kinase; LDH: Lactate Dehydrogenase; HBDH: Hydroxybutyrate Dehydrogenase; AST: Aspartate Aminotransferase; ALT: Alanine Transaminase; TP: Total Protein; ALB: Albumin; GLB: Globin; AGR: Albumin-to-Globin ratio; TBIL: Total Bilirubin; DBIL: Direct Bilirubin; IBIL: Indirect Bilirubin; Glu: Glucose; Cr: Creatinine; UA: Uric Acid; IgA: Immunoglobulin A; IgG: Immunoglobulin G; IgM: Immunoglobulin M; C3: Complement 3; CFB:

Complement factor B; CRP: C-reactive protein; PNI: Prognostic Nutritional Index; SII: Systemic ImmuneInflammation Score; AUC: area under receptor operating curve; Cl: confidence interval; OS: overall survival; C-index: Consistence index.

\section{Declarations}

\section{Ethics approval and consent to participate}

The ethical approval was obtained through the ethics committee of Cancer Hospital of Shantou University Medical College who surrendered the need for patient consent when the individual consents were not identified. And the research was conducted according to Declaration of Helsinki.

\section{Consent for publication}

None applicable.

\section{Availability of data and materials}

The datasets used and/or analysed during the current study are available from the corresponding author on reasonable request.

\section{Competing interests}

The authors declare that they have no competing interests.

\section{Funding}

This work was funded by grants from Natural Science Foundation of China (grant number 81972801); Guangdong Basic and Applied Basic Research Foundation (grants number 2019A1515011873 and 2018A0303130279); Science and Technology Planning Project of Shantou City (grants number 200605115266724 and 2017/166); Guangdong Esophageal Cancer Institute Science and Technology Program (grant number Q201906); and 2020 Li Ka Shing Foundation Cross-Disciplinary Research Grant (grant number 2020LKSFG01B).

\section{Authors Contributions}


Conceptualization: Can-Tong Liu, Yi-Wei Xu and Yu-Hui Peng; Methodology: Chao-Qun Hong and HaiPeng Guo; Formal analysis: Can-Tong Liu and Xu-Chun Huang; Resources: Hai-Peng Guo and Hong Guo; Validation: Xin-Yi Huang and Ling-Yu Chu; Investigation: Yi-Wei Lin and Lai-Feng Wei; Writing - original draft preparation: Can-Tong Liu and Chao-Qun Hong; Writing - review and editing: Can-Tong Liu and YiWei Xu; Funding acquisition: Chao-Qun Hong, Yi-Wei Xu and Yu-Hui Peng; Supervision: Yu-Hui Peng. All authors have read and agreed to the published version of the manuscript.

\section{Acknowledgements}

Not applicable.

\section{References}

1. Sung H, Ferlay J, Siegel RL, Laversanne M, Soerjomataram I, Jemal A, et al. Global cancer statistics 2020: GLOBOCAN estimates of incidence and mortality worldwide for 36 cancers in 185 countries. CA Cancer J Clin. 2021.doi:10.3322/caac.21660.

2. Siewert JR, Stein HJ. Classification of adenocarcinoma of the oesophagogastric junction. Br J Surg. 1998;85:1457-9.doi:10.1046/j.1365-2168.1998.00940.x.

3. Tran GD, Sun XD, Abnet CC, Fan JH, Dawsey SM, Dong ZW, et al. Prospective study of risk factors for esophageal and gastric cancers in the Linxian general population trial cohort in China. Int $\mathrm{J}$ Cancer. 2005;113:456-63.doi:10.1002/ijc.20616.

4. Haga $Y$, Hato S, Ikenaga M, Yamamoto $K$, Tsuburaya A, Doi K, et al. Validation of an assessment tool: Estimation of Postoperative Overall Survival for Gastric Cancer. Eur J Surg Oncol. 2018;44:51523.doi:10.1016/j.ejso.2018.01.002.

5. Okuda I, Kokubo T, Hoshihara Y, Udagawa H. [Imaging diagnosis of esophageal carcinoma by computed tomography and magnetic resonance imaging]. Nihon Geka Gakkai Zasshi. 2002;103:3316.

6. Kim HJ, Kim AY, Oh ST, Kim JS, Kim KW, Kim PN, et al. Gastric cancer staging at multi-detector row CT gastrography: comparison of transverse and volumetric CT scanning. Radiology. 2005;236:87985.doi:10.1148/radiol.2363041101.

7. Schroder W, Baldus SE, Monig SP, Beckurts TK, Dienes HP, Holscher AH. Lymph node staging of esophageal squamous cell carcinoma in patients with and without neoadjuvant radiochemotherapy: histomorphologic analysis. World J Surg. 2002;26:584-7.doi:10.1007/s00268-001-0271-5.

8. Reijnen C, IntHout J, Massuger L, Strobbe F, Kusters-Vandevelde HVN, Haldorsen IS, et al. Diagnostic Accuracy of Clinical Biomarkers for Preoperative Prediction of Lymph Node Metastasis in Endometrial Carcinoma: A Systematic Review and Meta-Analysis. Oncologist. 2019;24:e880e90.doi:10.1634/theoncologist.2019-0117.

9. Gao XL, Wang SS, Cao DB, Liu W. The role of plasma D-dimer levels for predicting lymph node and mediastinal lymph node involvement in non-small cell lung cancer. Clin Respir J. 2018;12:2151- 
6.doi:10.1111/crj.12786.

10. Meng ZW, Lin XQ, Zhu JH, Han SH, Chen YL. A nomogram to predict lymph node metastasis before resection in intrahepatic cholangiocarcinoma. J Surg Res. 2018;226:56-

63.doi:10.1016/j.jss.2018.01.024.

11. Zhang LX, Wei ZJ, Xu AM, Zang JH. Can the neutrophil-lymphocyte ratio and platelet-lymphocyte ratio be beneficial in predicting lymph node metastasis and promising prognostic markers of gastric cancer patients? Tumor maker retrospective study. Int J Surg. 2018;56:320-

7.doi:10.1016/j.ijsu.2018.06.037.

12. Kudou K, Nakashima Y, Haruta Y, Nambara S, Tsuda Y, Kusumoto E, et al. Comparison of Inflammation-Based Prognostic Scores Associated with the Prognostic Impact of Adenocarcinoma of Esophagogastric Junction and Upper Gastric Cancer. Ann Surg Oncol. 2020.doi:10.1245/s10434020-08821-y.

13. Jomrich G, Paireder M, Kristo I, Baierl A, Ilhan-Mutlu A, Preusser M, et al. High Systemic ImmuneInflammation Index is an Adverse Prognostic Factor for Patients With Gastroesophageal Adenocarcinoma. Ann Surg. 2019.doi:10.1097/SLA.0000000000003370.

14. Kudou K, Saeki H, Nakashima Y, Kamori T, Kawazoe T, Haruta Y, et al. C-reactive protein/albumin ratio is a poor prognostic factor of esophagogastric junction and upper gastric cancer. J Gastroenterol Hepatol. 2019;34:355-63.doi:10.1111/jgh.14442.

15. Rouzier R, Pusztai L, Garbay JR, Delaloge S, Hunt KK, Hortobagyi GN, et al. Development and validation of nomograms for predicting residual tumor size and the probability of successful conservative surgery with neoadjuvant chemotherapy for breast cancer. Cancer. 2006;107:145966.doi:10.1002/cncr.22177.

16. Huang YQ, Liang CH, He L, Tian J, Liang CS, Chen X, et al. Development and Validation of a Radiomics Nomogram for Preoperative Prediction of Lymph Node Metastasis in Colorectal Cancer. J Clin Oncol. 2016;34:2157-64.doi:10.1200/JC0.2015.65.9128.

17. Wang S, Yang L, Ci B, Maclean M, Gerber DE, Xiao G, et al. Development and Validation of a Nomogram Prognostic Model for SCLC Patients. J Thorac Oncol. 2018;13:133848.doi:10.1016/j.jtho.2018.05.037.

18. Kattan MW, Karpeh MS, Mazumdar M, Brennan MF. Postoperative nomogram for disease-specific survival after an RO resection for gastric carcinoma. J Clin Oncol. 2003;21:364750.doi:10.1200/JC0.2003.01.240.

19. Wen J, Yang Y, Liu P, Ye F, Tang H, Huang X, et al. Development and validation of a nomogram for predicting survival on the base of modified lymph node ratio in breast cancer patients. Breast. 2017;33:14-22.doi:10.1016/j.breast.2017.01.017.

20. Liu C-T, Hong C-Q, Huang X-C, Li E-M, Xu Y-W, Peng Y-H. Blood-based Markers in the Prognostic Prediction of Esophagogastric Junction Cancer. Journal of Cancer. 2020;11:433242.doi:10.7150/jca.44545. 
21. Amin MB, Edge SB, Greene FL, Byrd DR, Brookland RK, Washington MK, et al. AJCC Cancer Staging Manual2017.

22. Tibshirani R. Regression Shrinkage and Selection via the Lasso. Journal of the Royal Statistical Society Series B (Methodological). 1996;58:267-88.

23. Candido J, Hagemann T. Cancer-related inflammation. J Clin Immunol. 2013;33 Suppl 1:S7984.doi:10.1007/s10875-012-9847-0.

24. Chau I, Norman AR, Cunningham D, Waters JS, Oates J, Ross PJ. Multivariate prognostic factor analysis in locally advanced and metastatic esophago-gastric cancer-pooled analysis from three multicenter, randomized, controlled trials using individual patient data. J Clin Oncol. 2004;22:2395403.doi:10.1200/JC0.2004.08.154.

25. Koo DH, Ryoo BY, Kim HJ, Ryu MH, Lee SS, Moon JH, et al. A prognostic model in patients who receive chemotherapy for metastatic or recurrent gastric cancer: validation and comparison with previous models. Cancer Chemother Pharmacol. 2011;68:913-21.doi:10.1007/s00280-011-1561-8.

26. Takahari D, Boku N, Mizusawa J, Takashima A, Yamada Y, Yoshino T, et al. Determination of prognostic factors in Japanese patients with advanced gastric cancer using the data from a randomized controlled trial, Japan clinical oncology group 9912. Oncologist. 2014;19:35866.doi:10.1634/theoncologist.2013-0306.

27. Fuchs CS, Muro K, Tomasek J, Van Cutsem E, Cho JY, Oh SC, et al. Prognostic Factor Analysis of Overall Survival in Gastric Cancer from Two Phase III Studies of Second-line Ramucirumab (REGARD and RAINBOW) Using Pooled Patient Data. J Gastric Cancer. 2017;17:13244.doi:10.5230/jgc.2017.17.e16.

28. Horak ER, Leek R, Klenk N, LeJeune S, Smith K, Stuart N, et al. Angiogenesis, assessed by platelet/endothelial cell adhesion molecule antibodies, as indicator of node metastases and survival in breast cancer. Lancet. 1992;340:1120-4.doi:10.1016/0140-6736(92)93150-I.

29. Catal O, Ozer B, Sit M. Prediction of Lymph Node Metastasis in Colon Cancer via Platelet to Lymphocyte Ratio and Platelet Count. J Coll Physicians Surg Pak. 2020;30:2503.doi:10.29271/jcpsp.2020.03.250.

30. Ohuchi M, Inoue S, Ozaki Y, Ueda K. Platelet count and mean platelet volume are associated with not only bone, soft tissue, and lymph node metastases but also with malignant pleural effusion in lung cancer patients. Neoplasma. 2017;64:140-7.doi:10.4149/neo_2017_118.

31. Liu CT, Hong CQ, Huang XC, Li EM, Xu YW, Peng YH. Blood-based Markers in the Prognostic Prediction of Esophagogastric Junction Cancer. Journal of Cancer. 2020;11:433242.doi:10.7150/jca.44545.

32. Haemmerle M, Stone RL, Menter DG, Afshar-Kharghan V, Sood AK. The Platelet Lifeline to Cancer: Challenges and Opportunities. Cancer Cell. 2018;33:965-83.doi:10.1016/j.ccell.2018.03.002.

33. Namikawa T, Ishida N, Tsuda S, Fujisawa K, Munekage E, Iwabu J, et al. Prognostic significance of serum alkaline phosphatase and lactate dehydrogenase levels in patients with unresectable advanced gastric cancer. Gastric cancer : official journal of the International Gastric Cancer 
Association and the Japanese Gastric Cancer Association. 2019;22:684-91 doi:10.1007/s10120-0180897-8.

34. Lorente JA, Morote J, Raventos C, Encabo G, Valenzuela H. Clinical efficacy of bone alkaline phosphatase and prostate specific antigen in the diagnosis of bone metastasis in prostate cancer. $\mathrm{J}$ Urol. 1996;155:1348-51.

35. Kobayashi M, Okabayashi T, Sano T, Araki K. Metastatic bone cancer as a recurrence of early gastric cancer - characteristics and possible mechanisms. World J Gastroenterol. 2005;11:558791.doi:10.3748/wjg.v11.i36.5587.

36. Aminian A, Karimian F, Mirsharifı R, Alibakhshi A, Hasani SM, Dashti H, et al. Correlation of serum alkaline phosphatase with clinicopathological characteristics of patients with oesophageal cancer. East Mediterr Health J. 2011;17:862-6.doi:10.26719/2011.17.11.862.

37. Mori K, Janisch F, Parizi MK, Mostafaei H, Lysenko I, Enikeev DV, et al. Prognostic value of alkaline phosphatase in hormone-sensitive prostate cancer: a systematic review and meta-analysis. Int $\mathrm{J}$ Clin Oncol. 2020;25:247-57.doi:10.1007/s10147-019-01578-9.

38. Hao H, Chen L, Huang D, Ge J, Qiu Y, Hao L. Meta-analysis of alkaline phosphatase and prognosis for osteosarcoma. Eur J Cancer Care (Engl). 2017;26.doi:10.1111/ecc.12536.

39. Hu B, Yang XR, Xu Y, Sun YF, Sun C, Guo W, et al. Systemic immune-inflammation index predicts prognosis of patients after curative resection for hepatocellular carcinoma. Clin Cancer Res. 2014;20:6212-22.doi:10.1158/1078-0432.CCR-14-0442.

40. Zhang Y, Xiao G, Wang R. Clinical significance of systemic immune-inflammation index (SII) and Creactive protein-to-albumin ratio (CAR) in patients with esophageal cancer: a meta-analysis. Cancer Manag Res. 2019;11:4185-200.doi:10.2147/CMAR.S190006.

41. Qiu Y, Zhang Z, Chen Y. Prognostic Value of Pretreatment Systemic Immune-Inflammation Index in Gastric Cancer: A Meta-Analysis. Front Oncol. 2021;11:537140.doi:10.3389/fonc.2021.537140.

42. Ishdorj G, Streu E, Lambert P, Dhaliwal HS, Mahmud SM, Gibson SB, et al. IgA levels at diagnosis predict for infections, time to treatment, and survival in chronic lymphocytic leukemia. Blood Adv. 2019;3:2188-98.doi:10.1182/bloodadvances.2018026591.

43. Yuan K, Ye J, Liu Z, Ren Y, He W, Xu J, et al. Complement C3 overexpression activates JAK2/STAT3 pathway and correlates with gastric cancer progression. J Exp Clin Cancer Res. 2020;39:9.doi:10.1186/s13046-019-1514-3.

44. Kim SH, Lee MJ, Hwang HK, Lee SH, Kim H, Paik YK, et al. Prognostic potential of the preoperative plasma complement factor $B$ in resected pancreatic cancer: A pilot study. Cancer biomarkers : section A of Disease markers. 2019;24:335-42.doi:10.3233/CBM-181847.

45. Lee MJ, Na K, Jeong SK, Lim JS, Kim SA, Lee MJ, et al. Identification of human complement factor B as a novel biomarker candidate for pancreatic ductal adenocarcinoma. J Proteome Res. 2014;13:4878-88.doi:10.1021/pr5002719.

46. Tokunaga R, Imamura Y, Nakamura K, Uchihara T, Ishimoto T, Nakagawa S, et al. Carbohydrate antigen 19-9 is a useful prognostic marker in esophagogastric junction adenocarcinoma. Cancer 
medicine. 2015;4:1659-66.doi:10.1002/cam4.514.

Figures

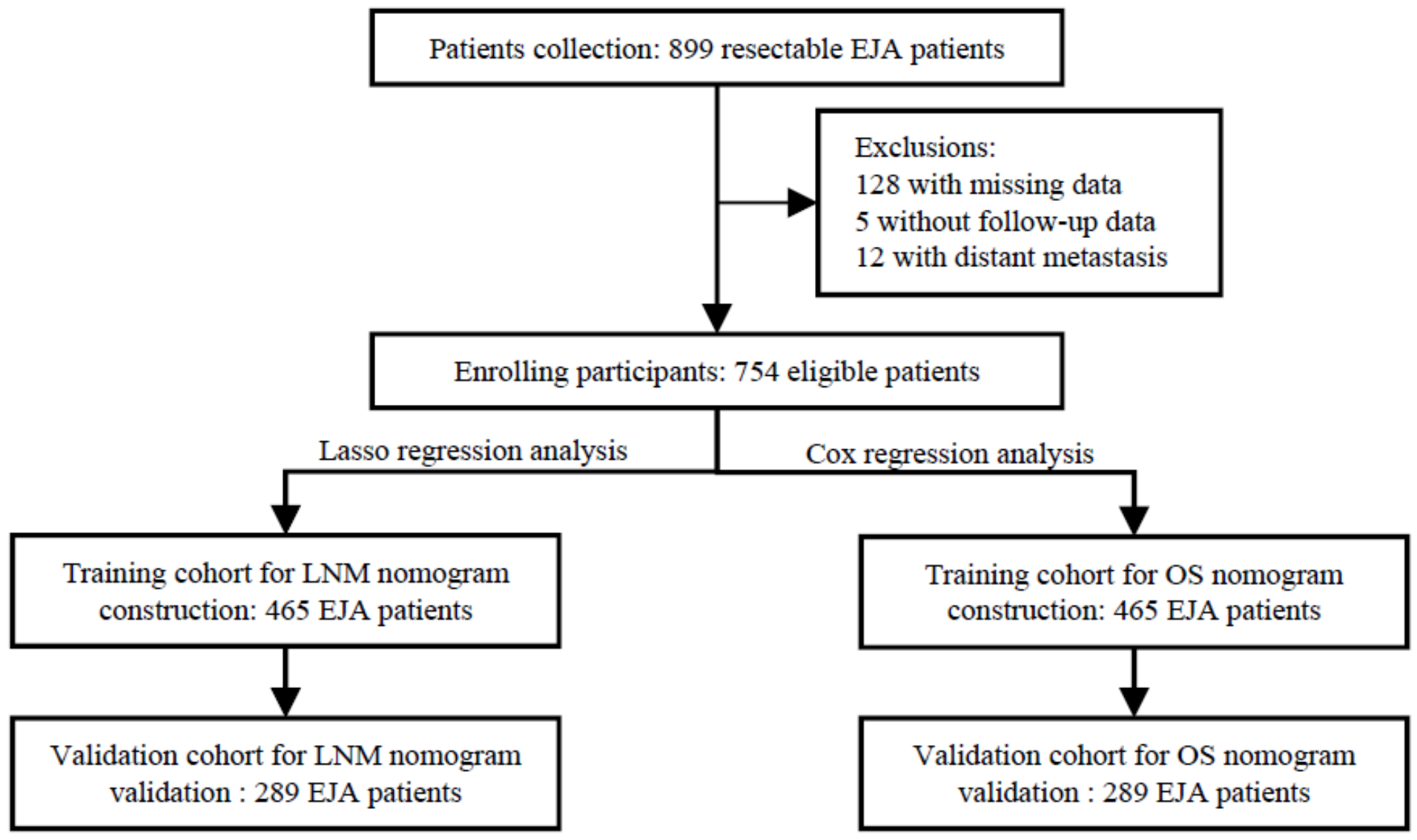

Figure 1

Recruitment process for EJA patients

EJA: esophagogastric junction adenocarcinoma; LNM: lymph node metastasis; OS: overall survival 

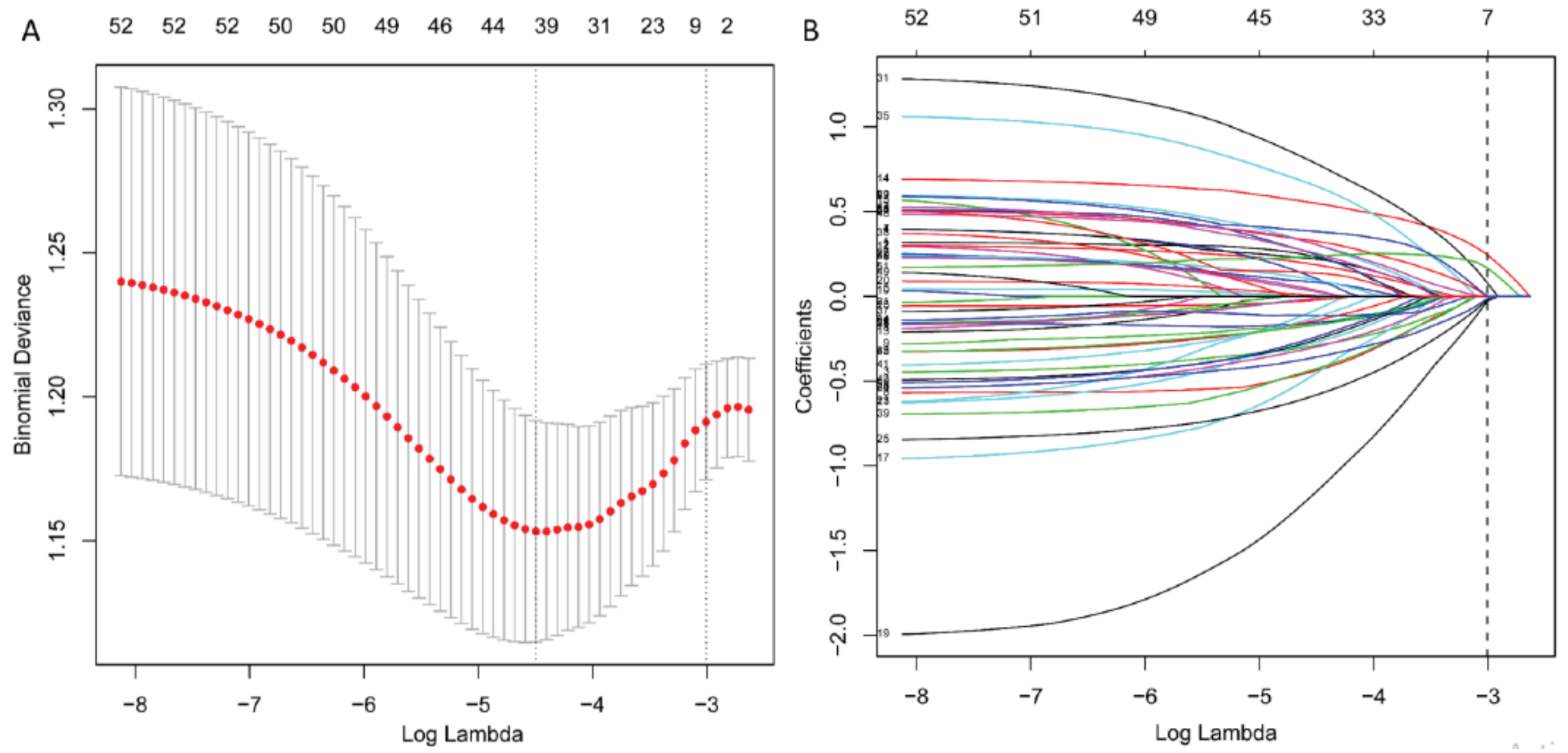

Figure 2

Lasso regression analysis of parameters selection for lymph node metastasis nomogram building

(A) $\lambda$ selection in the Lasso logistic regression model used 10 -fold cross-validation. Dotted vertical lines were drawn at the optimal values by using the minimum criteria and the 1 standard error. $\lambda$ value of 0.049 with $\log (\lambda)$ of -3.016 was chosen. (B) Lasso coefficient profiles of the 52 variables. Coefficient profile plot was produced against the $\log (\lambda)$ sequence. Vertical line was drawn at the value where $\lambda$ resulted in 7 nonzero coefficients. 

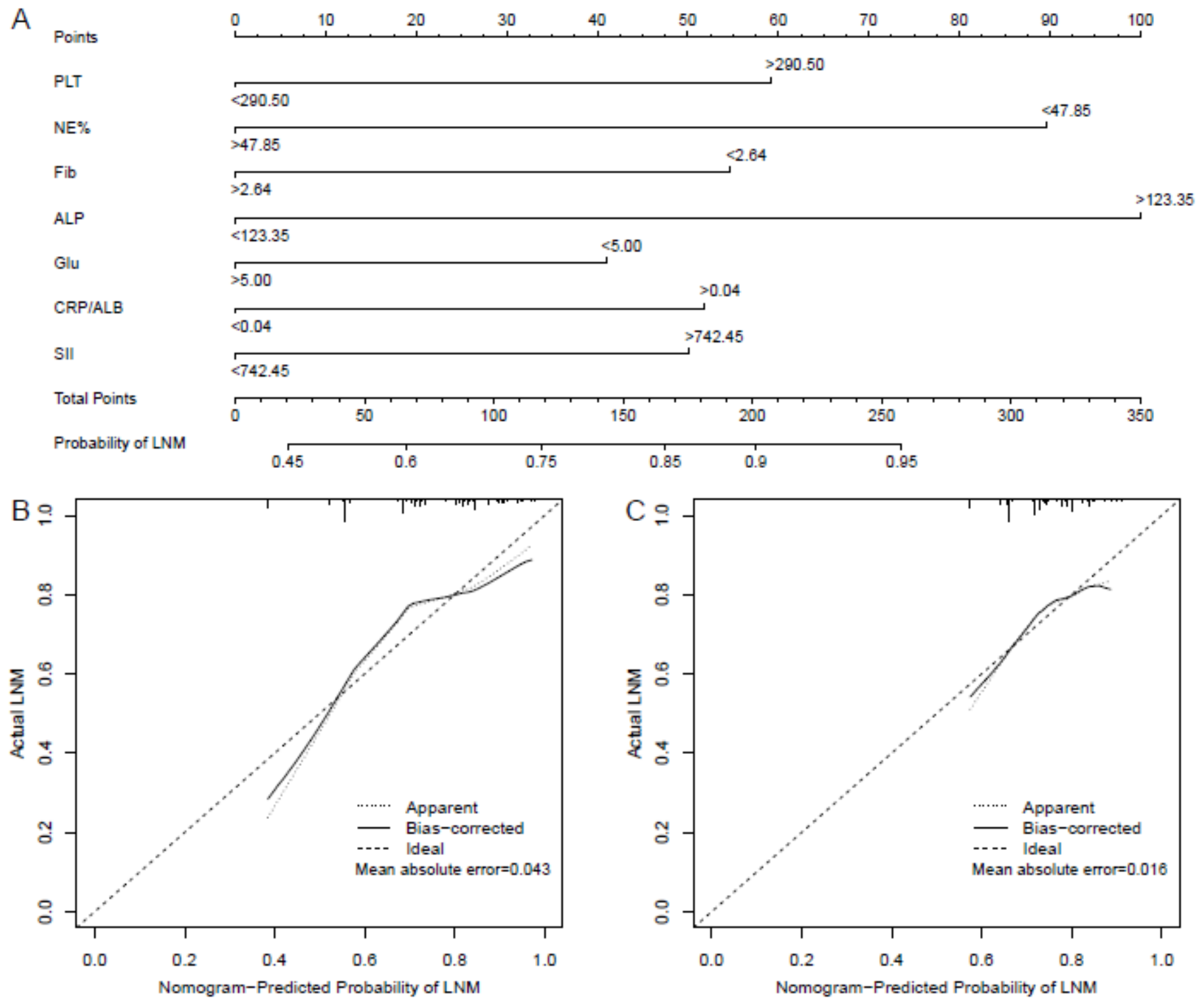

Figure 3

Nomogramand calibration curves for LNM prediction of EJA patients

(A) nomogram; (B) for training cohort; (C) for validation cohort 


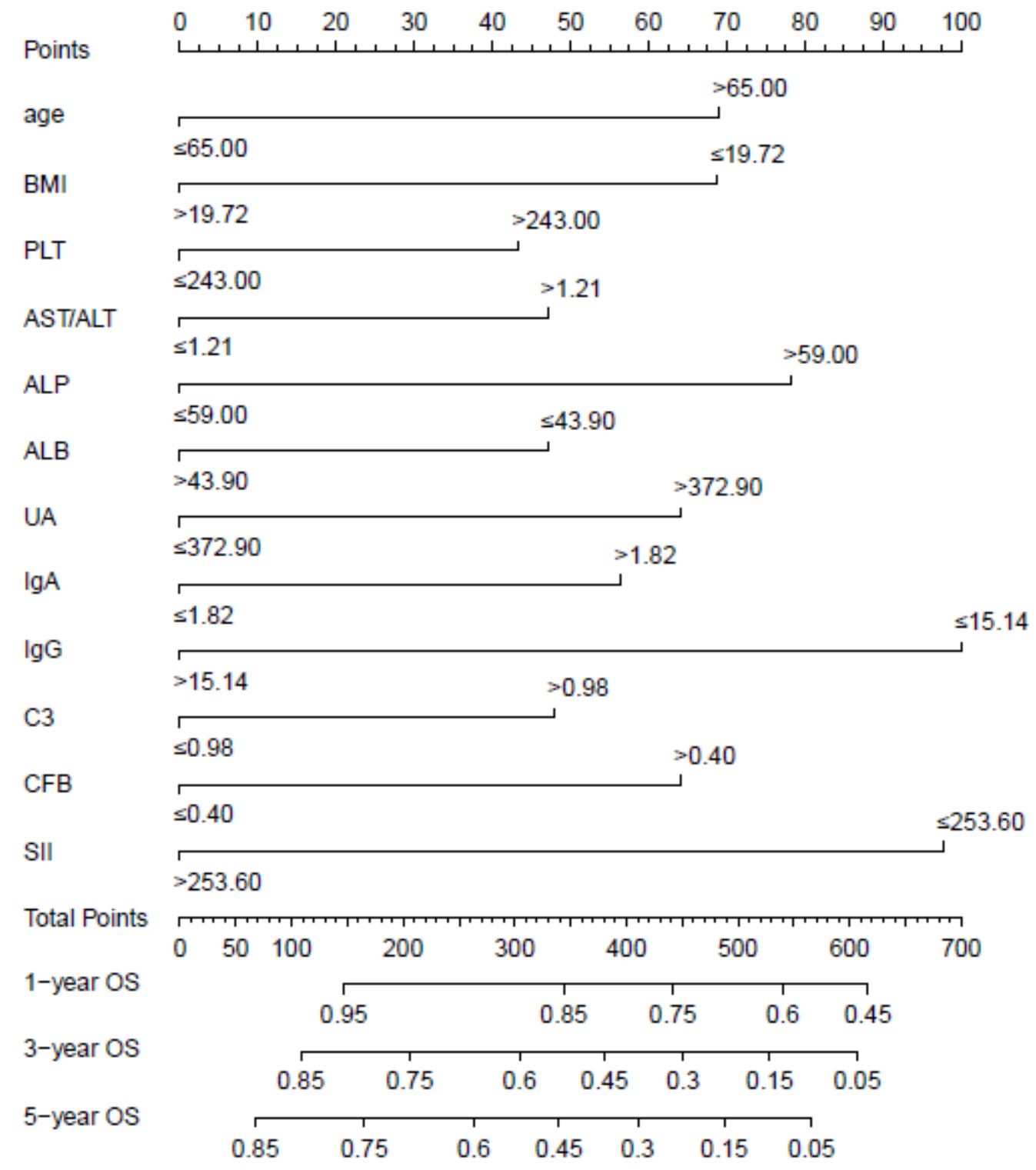

Figure 4

Nomogram for predicting OS of EJA patients. 

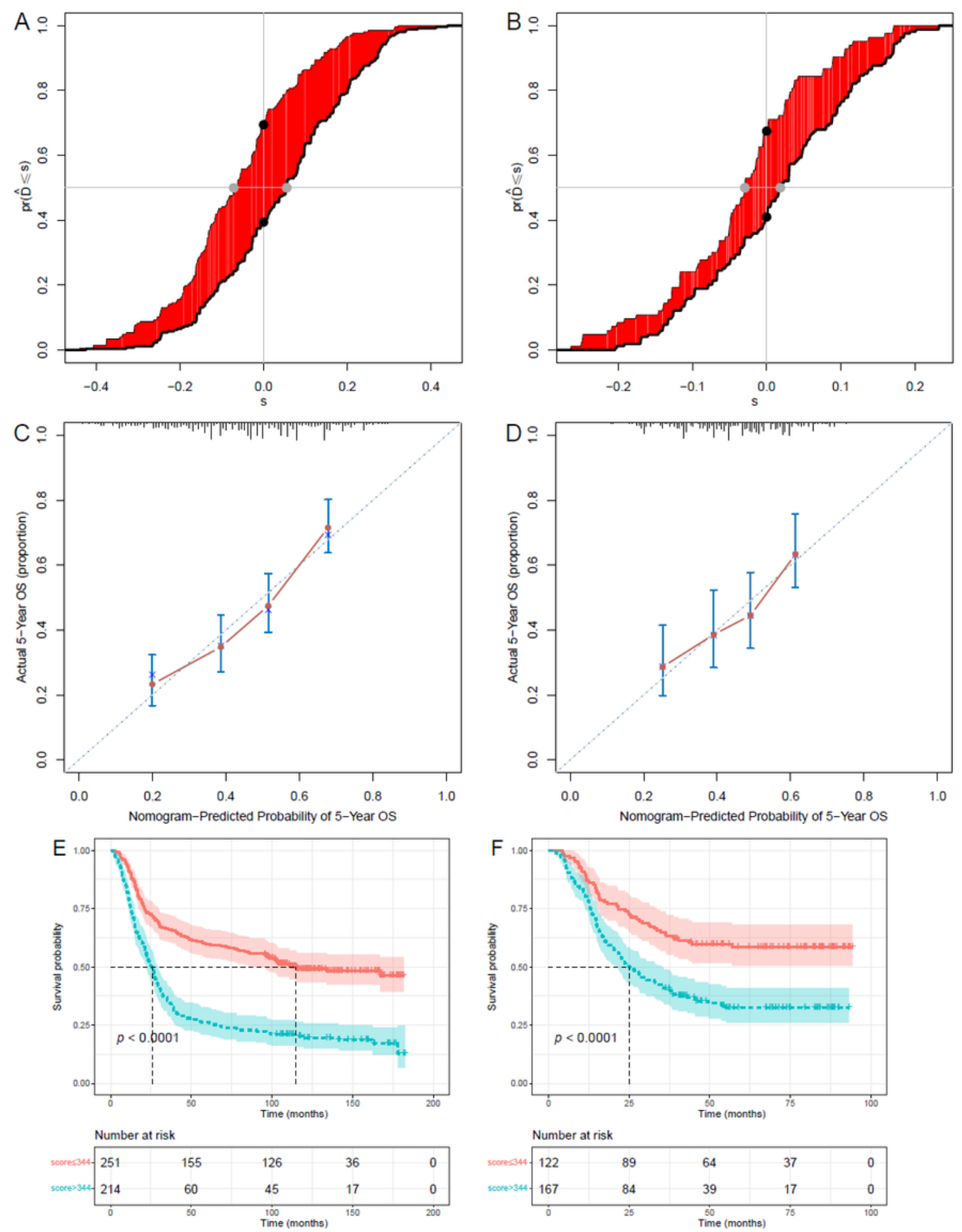

\section{Figure 5}

\section{Assessment of OS nomogram}

(A, B) The IDI and NRI of the combination of OS nomogram and TNM system in predicting 5-year OS of EJA patientsfor the training and validation cohort. The red area is IDI, the added predicted value. The distance between the two black dots represents NRI.(C, D) Calibration curves were plotted for the training 
and validation cohort. (E, F) Survival curves were plotted using Kaplan-Meier survival analysis and compared by the log-rank test based on the training and validation cohort.

\section{Supplementary Files}

This is a list of supplementary files associated with this preprint. Click to download.

- SupplementaryMaterials.docx 